\title{
Récupération d'énergie hydraulique : petites installations d'énergie associées à de grands projets hydrauliques
}

\section{Recovery of hydraulic energy : small installations associated with major hydraulic projects}

J. P. Rouyer

EDF
C. Le Plomb

CNR
Le cadre juridique des petites installations hydroélectriques

Le cadre juridique des petites installations est celui, plus général, défini par la loi du 16 octobre 1919 qui stipule que "nul ne peut disposer de l'énergie des marées, des lacs et des cours d'eau quel que soit leur classement, sans concession ou autorisation de l'Etat".

La limite entre les régimes de la concession ou de l'autorisation vient d'être portée (loi du 15 juillet 1980) à $4500 \mathrm{~kW}$ (il s'agit de la puissance maximale brute, correspondant à une puissance électrique nette de l'ordre de $3700 \mathrm{~kW})$.

Les ouvrages de récupération d'énergie proprement dite s'inscrivent dans les trois cas suivants :

- ils sont construits en même temps qu'un ouvrage hydroélectrique plus important : la concession couvre alors l'ensemble des installations ;

- ils sont ajoutés, après coup, à un ouvrage hydroélectrique existant : un avenant à la concession d'origine peut être alors demandé ;

- ils sont ajoutés à un ouvrage n'ayant pas fait l'objet d'une concession au titre de la loi 1919 : une concession ou une autorisation spécifiques sont alors nécessaires.

Les petites installations de récupération d'énergie

\section{Leur définition}

L'originalité de ces installations réside uniquement dans la liaison hydraulique aux ouvrages plus importants auxquels elles sont associées; nous verrons à ce sujet, dans les exemples de réalisation dont il sera question plus loin, que cette liaison prend généralement deux formes :

\section{1) Les groupes de restitution ou de récupération.}

Leur existence est liée soit à la nécessité de restituer dans la rivière une part (débit réservé) du débit qui passait là avant la réalisation d'un ouvrage de retenue, soit à l'existence d'un débit imposé pour une régularisation de rivière ou par une alimentation d'ouvrages situés plus en aval (ouvrages hydroélectriques ou autres).

Suivant la façon dont la consigne de débit réservé est élaborée, deux cas sont à considérer ; dans le quatrième paragraphe de cet exposé, on reviendra en détail sur cette distinction et les considérations économiques qui y sont attachées.

2) Les groupes de production installés en compléments d'ouvrages hydrauliques préexistants.

Cette fois, la démarche est différente : un site hydraulique existe, déjà équipé pour d'autres buts que la production d'énergie, pour la navigation par exemple.

L'étude technique et économique porte alors de façon classique sur la recherche d'une optimisation des installations conduisant à un enrichissement maximal (l'enrichissement étant défini comme la différence entre les produits et les charges).

\section{Le programme d'Electricité de France (EDF) et de la Compagnie Nationale du Rhône (CNR).}

Des groupes de restitution ont déjà été construits dans le passé ; une étude réalisée en 1977 par M. Cotillon recensait en effet pour EDF :

- pour les petites machines de puissance inférieure à $2000 \mathrm{~kW}$ : 15 groupes de restitution sur un total de 277 unités :

- pour les petites machines de puissance supérieure à $2000 \mathrm{~kW}: 10$ groupes de restitution sur un total de 151 unités.

Les nouvelles valorisations de l'énergie qui résultent des crises pétrolières successives ont permis de justifier 
l'intérêt économique d'installations nouvelles associées à des aménagements hydroélectriques existants, "l'enrichissement" défini plus haut devenant positif : c'est ainsi que depuis 1975 ont été installés par EDF 6 groupes supplémentaires apportant une puissance totale de $7800 \mathrm{~kW}$ et une production de 29 millions de $\mathrm{kWh}$. La CNR, de son côté, étudie l'installation de groupes de récupération supplémentaires sur le Rhône à l'aval de Lyon; ces projets portent pour le moment sur une puissance de $3000 \mathrm{~kW}$ et une production de 24 millions de $\mathrm{kWh}$.

En outre, les études d'impact réalisées à l'occasion de la construction de nouveaux ouvrages amènent organismes de tutelle et maîtres d'ouvrage à considérer avec une attention accrue diverses contraintes liées à la protection de l'environnement ; c'est ainsi que la CNR s'est engagée, à l'occasion de l'aménagement des chutes du Haut-Rhône, à restituer dans le lit du fleuve des débits importants relativement au débit d'équipement. Cela conduit, pour les chutes de Chautagne, Belley et Brégnier-Cordon, à l'installation de 5 groupes apportant une puissance de $6500 \mathrm{~kW}$ et une production de 48 millions de $\mathrm{kWh}$, dont les mises en service s'échelonneront de 1981 à 1984.

En complément à ces groupes de restitution ou de récupération, des études sont en cours pour l'équipement en groupes de production d'autres ouvrages hydrauliques et notamment des ouvrages de navigation. Le projet le plus avancé, et semble-t-il le plus important par sa production (180 millions de $\mathrm{kWh}$ pour une puissance installée de $32000 \mathrm{~kW}$ ) est celui de l'équipement par EDF de la Moselle canalisée. Il est exposé ci-après.

L'ensemble des réalisations récentes et des projets d'EDF et de la CNR représente au total une production de 280 millions de $\mathrm{kWh}$ et une puissance de près de $50000 \mathrm{~kW}$ qui peuvent paraitre faibles au regard de la production hydroélectrique totale (actuellement $63,9 \mathrm{TWh})$; mais s'agissant d'une énergie nationale et renouvelable, elle n'est pas à dédaigner dans les difficiles circonstances énergétiques actuelles.

\section{Quelques réalisations récentes d'EDF}

\section{Aménagement de l'usine hydroélectrique de Nèpes sur la Cère}

Le barrage de compensation de Nèpes est situé sur la Cère à l'aval du barrage de St-Etienne Cantalès et à environ $1 \mathrm{~km}$ à l'amont de Laroquebrou dans le département du Cantal (Fig. 1).

Il comportait en rive droite deux conduites de restitution de 2,30 $\mathrm{m}$ de diamètre équipées chacune d'une vanne papillon permettant de régler le débit restitué et d'effectuer des lâchures turbinées à l'aval par les usines de Lamativie et de Laval-de-Cère I.

Le nouvel aménagement permet de valoriser ces lâchures par l'installation, immédiatement à l'aval, d'un groupe de restitution de $2800 \mathrm{~kW}$ comprenant une turbine Kaplan à distributeur fixe et pales variables, accouplée à une génératrice asynchrone. Le gain de productibilité est de 8 millions de $\mathrm{kWh}$.

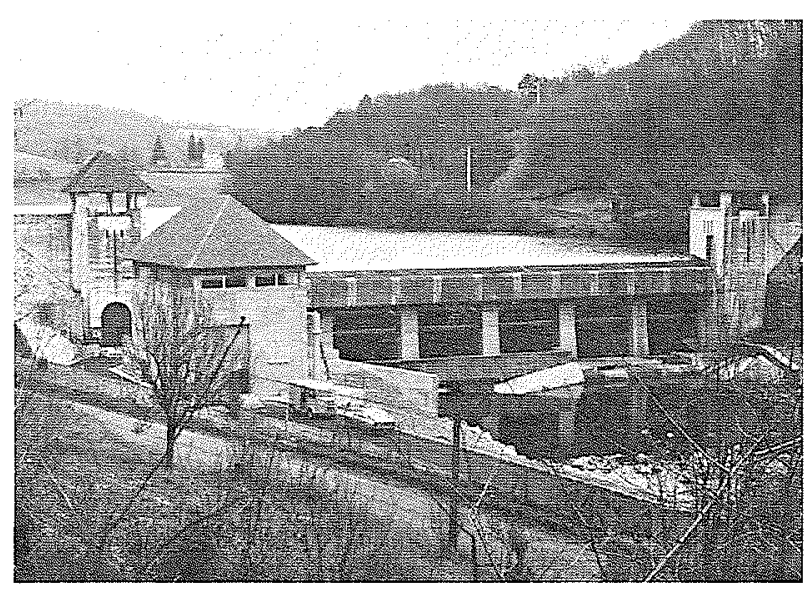

Figure 1 -.. Usine de Nèpes - Vue aval rive droite.

Le débit équipé est de $27,5 \mathrm{~m}^{3} / \mathrm{s}$; la hauteur de chute brute maximale (pour $\mathrm{Q}=27,5 \mathrm{~m}^{3} / \mathrm{s}$ ) est de $13 \mathrm{~m}$ et la hauteur de chute nette de $12,20 \mathrm{~m}$.

La turbine a une vitesse de rotation de $250 \mathrm{tr} / \mathrm{mn}$; elle a été fabriquée par les Etablissements Bouvier à Grenoble ; la génératrice asynchrone a été construite par les Etablissements Jeumont-Schneider.

Les travaux ont commencé en juillet 1977 et la mise en service est intervenue en février 1979 ; le coût des travaux et fournitures, hors taxes, sur les bases économiques de janvier 1976, s'est élevé à 3,2 MF pour le génie civil et $4,3 \mathrm{MF}$ pour les travaux d'électromécanique.

\section{L'usine de Lamanon, près de Salon de Provence (Fig. 2).}

Un débit prélevé sur le canal industriel de l'usine hydroélectrique de Salon, réalimente des canaux agricoles (notamment ceux de Craponne et des Alpines Méridionales et Septentrionales) ; les travaux d'aménagement ont consisté à construire une usine pour turbiner ce débit.

La conduite existante en béton, enterrée, a une longueur de $460 \mathrm{~m}$ et un diamètre de $3,50 \mathrm{~m}$; les ouvrages d'amenée complémentaires construits sont 2 conduites enterrées, piquées sur la conduite existante, et constituées par 2 tuyaux métalliques enrobés de béton, dont le diamètre intérieur est de $2,70 \mathrm{~m}$ et les longueurs respectivement de $18,2 \mathrm{~m}$ et $15,6 \mathrm{~m}$.

L'usine comporte deux turbines Kaplan horizontales de puissance unitaire égale à $1145 \mathrm{~kW}$ et prévues chacune pour un débit de $17,5 \mathrm{~m}^{3} / \mathrm{s}$ (la hauteur de chute brute maximale est de $7,50 \mathrm{~m}$ et la chute nette maximale à plein débit est de $5,50 \mathrm{~m}$ ) ; leur vitesse de rotation est de $167 \mathrm{tr} / \mathrm{min}$ et elles sont associées à 2 génératrices asynchrones par l'intermédiaire de multiplicateurs $167 / 757 \mathrm{tr} / \mathrm{min}$.

La puissance maximale disponible est de $1625 \mathrm{~kW}$ et la production annuelle moyenne est de 8,7 millions de $\mathrm{kWh}$.

Les turbines ont été fabriquées par la Société Dumont à St-Uze (Drôme) et les génératrices par CEM. Les travaux ont commencé en 1978 et la mise en service est intervenue en octobre 1979 ; le coût des travaux s'est 


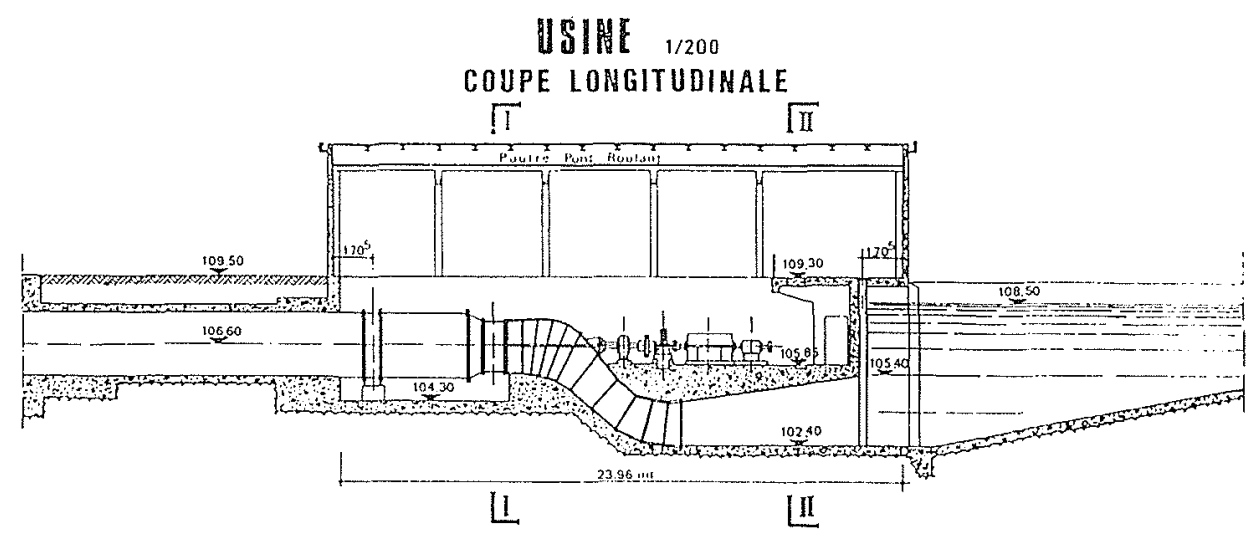

PLAN GOUPE

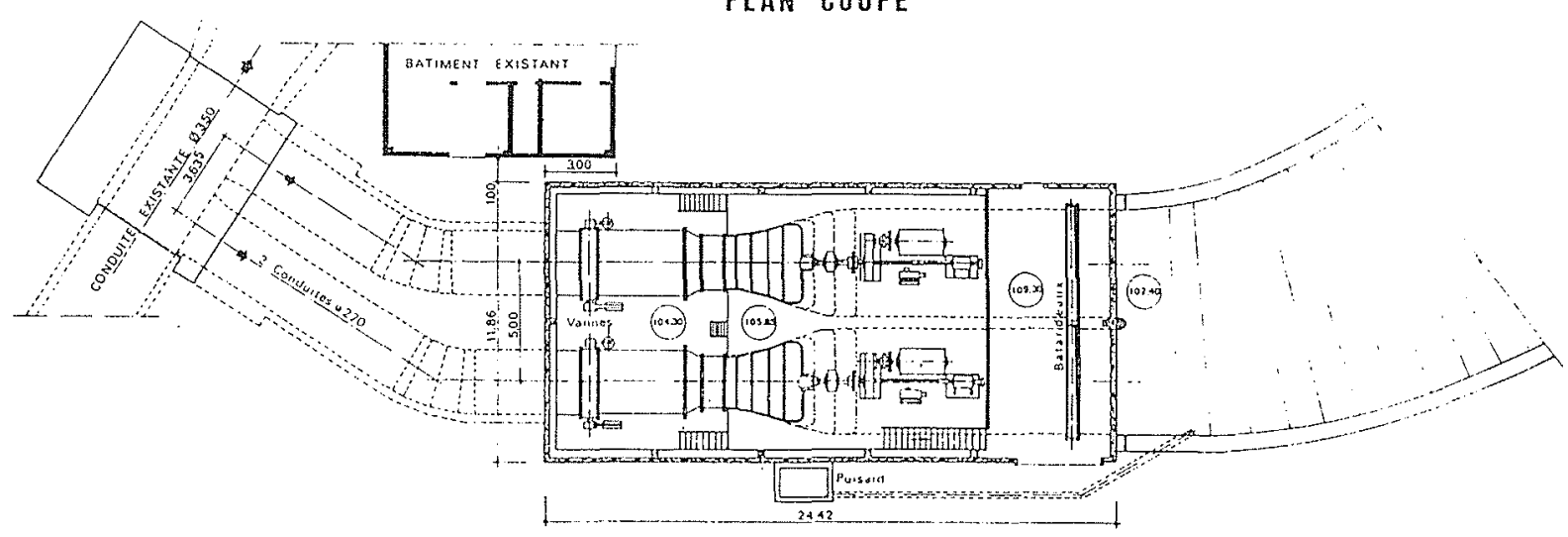

Figure 2 -- Usine de Lamanon -- Coupe longitudinale et vue en plan.

élevé à 5,5 MF (dont 1,5 MF pour le génie civil) sur les bases économiques de janvier 1977.

\section{Le groupe de restitution de Strasbourg (Fig. 3).}

Ce groupe est installé en rive gauche des passes mobiles du barrage de Strasbourg sur le Rhin, l'ensemble de l'ouvrage contournant la culée du barrage.

Il turbine le débit de $15 \mathrm{~m}^{3} / \mathrm{s}$ qui doit être restitué en permanence en aval.

Les ouvrages d'amenée comportent notamment une conduite forcée Bonna à âme tôle, de diamètre intérieur égal à $2,5 \mathrm{~m}$ et dont la longueur est de $39 \mathrm{~m}$; elle est noyée dans le remblai.

La chute brute nominale est de $8,25 \mathrm{~m}$; la turbine du type hélice à distributeur fixe (constructeur : Dumont), a 5 pales réglables à l'arrêt ; sa puissance nominale est de $1020 \mathrm{~kW}$ et sa vitesse de rotation de $253 \mathrm{tr} / \mathrm{min}$. Elle entraîne directement une génératrice asynchrone (constructeur : CEM). La production annuelle est de près de 8 millions de $\mathrm{kWh}$.

Les travaux ont débuté en 1978 et la mise en service a eu lieu en mars 1980 ; le coût total est de l'ordre de 10 millions de francs, sur les bases économiques de janvier 1979.

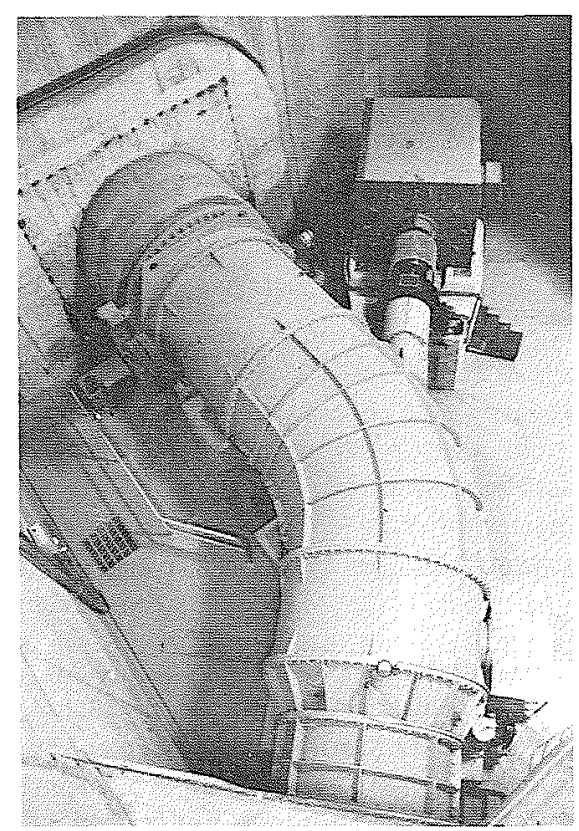

Figure 3 - Usine de Strasbourg - la turbine et le groupe générateur. 


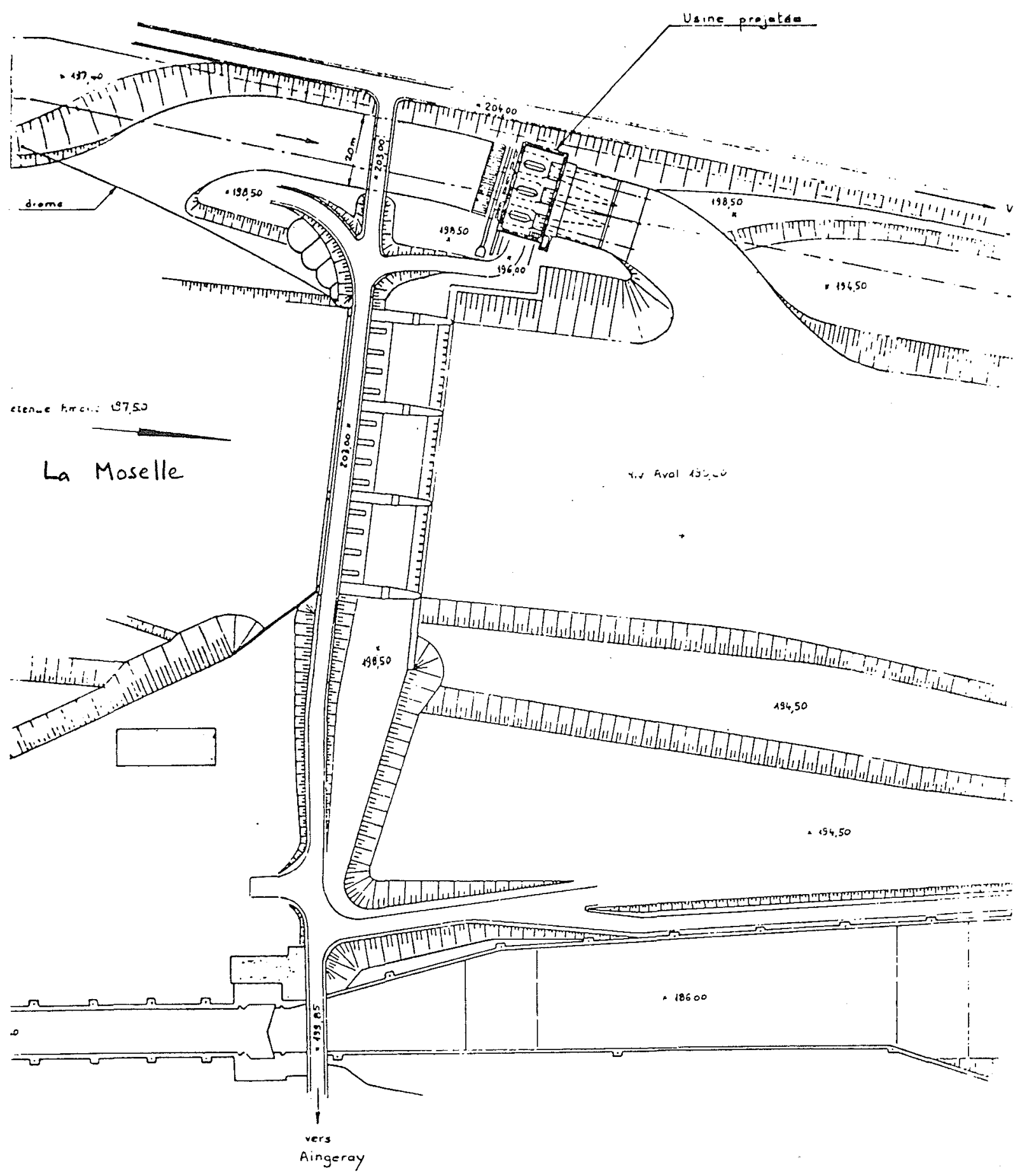

Figure 4 - Equipement de la Moselle - implantation projetée pour l'usine de Liverdun.

\section{Aménagement d'ouvrages hydroélectriques sur la Moselle (Fig. 4).}

L'étude actuellement entreprise par le Groupe Régional de Production Hydraulique Rhin d'EDF concerne la partie comprise entre Neuves-Maisons, au sud-ouest de Nancy, jusqu'à Apach, à la frontière avec le Luxembourg et la République Fédérale d'Allemagne, soit environ $130 \mathrm{~km}$.

Cette partie de la Moselle est canalisée; elle est ouverte à la navigation à grand gabarit et donc accessible aux bateaux de $1500 \mathrm{t}$ ayant un tirant d'eau de $2,50 \mathrm{~m}$ et aux convois poussés de $3000 \mathrm{t}$.

Cette mise au gabarit européen a nécessité la construction ou l'aménagement de nombreux ouvrages entre 1960 et 1978 ; on compte ainsi 17 écluses et 14 barrages.

Les caractéristiques hydrologiques de la Moselle permettent d'envisager l'équipement hydroélectrique d'un certain nombre de sites liés à des barrages existants ; le débit moyen annuel évolue d'amont en aval 
de $68 \mathrm{~m}^{3} / \mathrm{s}$ en amont du confluent avec la Meurthe jusqu'à $135 \mathrm{~m}^{3} / \mathrm{s}$ à la frontière; les plus forts débits sont en hiver ; la dénivellation du tronçon de $130 \mathrm{~km}$ est de $73 \mathrm{~m}$.

L'ensemble des aménagements représenterait une puissance totale maximale possible de l'ordre de $32000 \mathrm{~kW}$, pour un productible moyen annuel d'environ 180 millions de $\mathrm{kWh}$, dont 95 millions en hiver. Les débits équipés varieront de $90 \mathrm{~m}^{3} / \mathrm{s}$ à $150 \mathrm{~m}^{3} / \mathrm{s}$ et les chutes nettes de $2,50 \mathrm{~m}$ à $7 \mathrm{~m}$. La puissance des usines serait variable de $1800 \mathrm{~kW}$ à $5000 \mathrm{~kW}$; environ 25 groupes de production sont prévus.

La réalisation des dix usines envisagées pourrait s'échelonner de 1982 à 1988 environ, par exemple à raison de deux par an ; les premières mises en service pourraient intervenir en 1983 ou 1984. Le montant total des travaux est estimé à $250 \mathrm{MF}$, aux conditions économiques de janvier 1980.

\section{Analyse économique des projets}

En cherchant à analyser les conditions dans lesquelles est étudiée l'installation de groupes de récupération associés à des grands projets hydrauliques, on pourrait être tenté de distinguer, les cas où l'objectif est la production d'énergie électrique (projets hydroélectriques "purs" ou projets à buts multiples avec production électrique) et ceux n'ayant pas cet objectif.

En fait, si une telle distinction peut s'imposer au plan administratif, elle a peu de raisons d'être du point de vue économique, et encore moins du point de vue technique.

S'il s'agit du projet d'un nouvel aménagement, les performances économiques respectives de l'équipement principal et de l'installation associée peuvent conduire à diverses situations. Deux d'entre elles sont favorables à la réalisation d'un équipement de récupération :

- l'équipement principal est rentable en lui-même, et l'installation associée rentable, elle aussi, améliore le bilan global du projet :

- l'équipement principal présente une rentabilité incertaine et l'installation associée permet au projet d'être globalement rentable.

S'il s'agit de compléter un aménagement hydraulique existant et quel qu'en ait été l'objectif initial, il est bien évident que la seule rentabilité de l'installation associée sera prise en compte.

Les exemples qui sont présentés plus loin montrent que la définition de l'équipement de récupération proprement dit peut revêtir deux aspects principalement conditionnés par la consigne de débit réservé.

- ou bien cette consigne, c'est-à-dire le programme des débits à restituer en fonction du temps, est simple (constant toute l'année. ou à 2 valeurs de durée semestrielle) et son point d'application est situé à l'aval immédiat de l'ouvrage de retenue :

- ou bien elle comporte 3, voire 4 paliers de débit dans l'année et son point d'application se trouve en un lieu qui est influencé par des apports extérieurs assez nettement variables.
- Dans le premier cas, le débit de consigne est facile à réaliser. Les modulations sont obtenues par démarrage ou arrêt d'un ou deux groupes dont le débit unitaire, fixe, est déterminé en fonction de la consigne $\left({ }^{1}\right)$.

Ce fut le cas sur le Bas-Rhône pour Charmes, PierreBénite et St-Pierre de Bœuf; plus récemment c'est le cas de Chautagne sur le Haut-Rhône, Strasbourg sur le Rhin. La rentabilité de l'installation, généralement facile à obtenir (compte tenu de la régularité des débits) est simplement vérifiée.

En fait l'équipement optimal se situe le plus souvent à l'une des bornes des paliers de débit considérés.

- Dans le second cas, l'utilisation d'un ou deux groupes sans réglage, s'adaptant assez mal aux modulations fines du débit, risque de conduire à un équipement peu ou pas rentable. On admet, alors, d'utiliser un équipement plus perfectionné, avec turbine réglable en débit, ou d'associer le barrage à la fonction de restitution au moyen des clapets dont sont équipées certaines de ses vannes. L'optimum d'équipement se trouve, alors, en un point de l'intervalle qui ne coïncide pas forcément avec l'une des bornes et il s'agit de définir les dimensions optimales du ou des groupes de récupération à installer. C'est la situation dans laquelle nous nous sommes trouvés à Belley et, tout récemment, à Bregnier Cordon. C'est aussi celle des aménagements de navigation, d'irrigation (Lamanon) ou de compensation (Nèpes).

La méthode consiste à rechercher l'enrichissement $\left({ }^{2}\right)$ maximal.

\section{Un exemple d'optimisation : Brégnier-Cordon sur le Haut-Rhône à l'aval de Belley}

Le schéma général de la chute avec indication du point d'application de la consigne, fait l'objet de la figure 5 .

Une première approche prenant en compte les débits réservés moyens mensuels de la consigne, d'une part, et du Guiers, d'autre part, a permis de déterminer les débits moyens mensuels à restituer (Fig. 6).

L'optimisation a été conduite à partir des débits moyens journaliers, sur la base d'un équipement comportant deux machines identiques. Du type Bulbe "amont", réglables en débit, avec multiplicateur et génératrice asynchrone rapide, ces machines appartiennent à une gamme dont les éléments techniques et commerciaux sont facilement accessibles sur catalogue.

Ce matériel, de construction modulaire standardisée, couvre largement le domaine d'optimisation (de 3 à $20 \mathrm{~m}$ en chute et de 5 à $70 \mathrm{~m}^{3} / \mathrm{s}$ en débit). Pour les chutes existantes au barrage et le débit approximatif considéré, cinq possibilités ont été retenues (Fig. 7). Les éléments économiques (Actif hydraulique, investissements, etc.) ont été calculés.

(1) Un ou plusieurs groupes, réglables à l'arrêt, peuvent aussi être utilisés.

(2) Au sens de la Note Bleue 


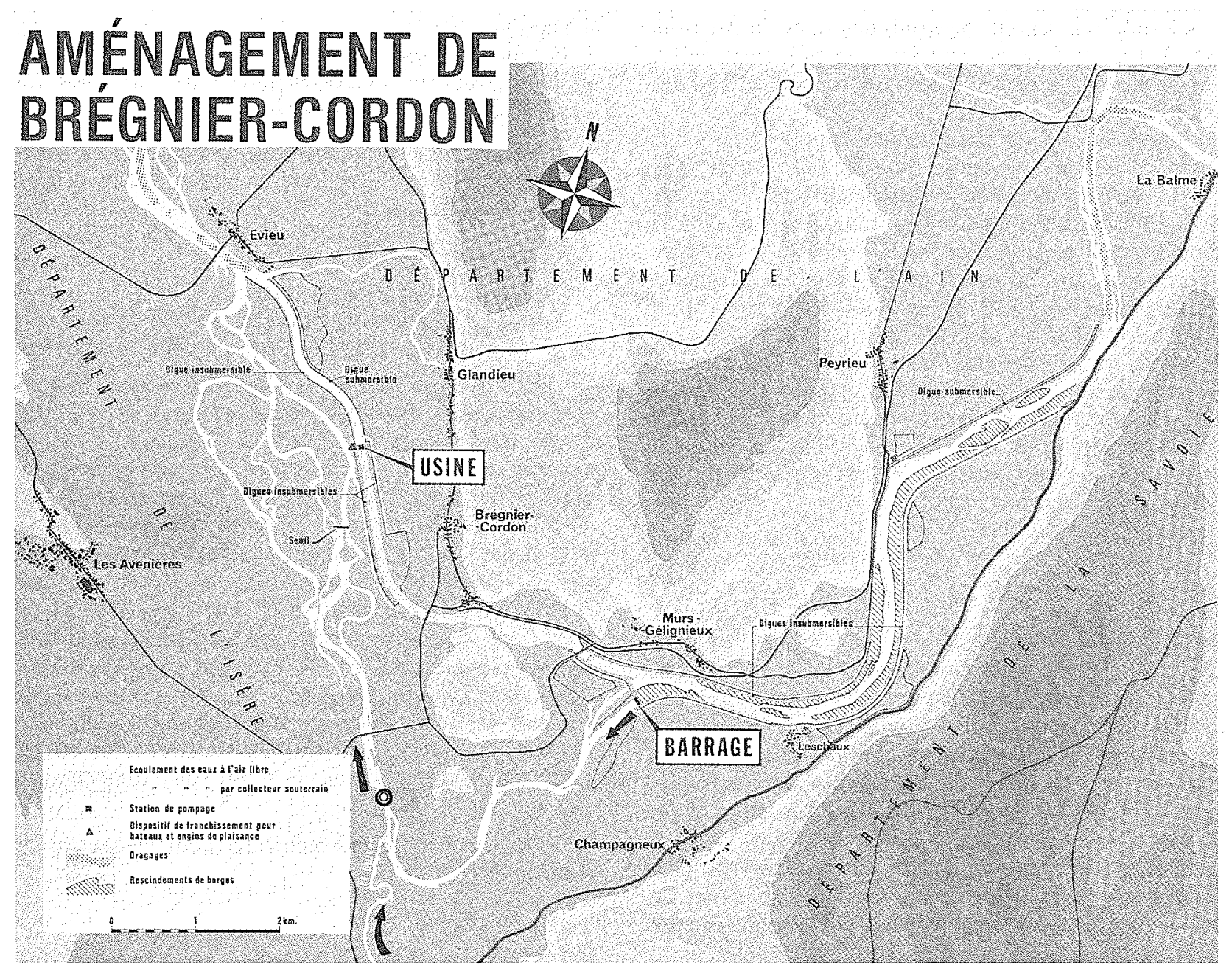

Figure 5 - Bregnier Cordon - Schéma général de l'aménagement.

Les résultats obtenus (Fig. 8) appellent un certain nombre de remarques:

- la rentabilité propre de l'installation est assurée dans tous les cas,

- la machine couvrant les débits compris entre 34 et $42 \mathrm{~m}^{3} / \mathrm{s}$ (68 et $84 \mathrm{~m}^{3} / \mathrm{s}$ pour l'ensemble de l'équipement) représente le choix optimal,

- les variations d'enrichissement de part et d'autre de cet optimum ne sont pas extrêmement sensibles.

En conclusion, la recherche de l'enrichissement maximal confirme l'approche faite à partir de l'examen des valeurs moyennes des débits de la rentabilité de l'installation de récupération.

La marge d'incertitude inhérente aux calculs réalisés, jointe dans ce cas précis, à la sensibilité assez limitée de l'optimisation, font que le choix final pourra, dans les limites du domaine exploré, être influencé par certains éléments extérieurs, (différences concernant la technologie du matériel, ou la conception des gammes proposées par les différents constructeurs, recherche d'une standardisation entre plusieurs aménagements voisins exploités conjointement, etc.).

\section{Réalisations et projets CNR}

Au total, sur huit sites du Rhône $\left({ }^{3}\right)$, dix machines totaliseront, fin 1983, une puissance de $11,9 \mathrm{MW}$ et produiront en année moyenne $90 \mathrm{GWh}$. Le détail en est donné par le tableau de la figure 9 .

Leur implantation dans l'une des piles-culées du barrage constitue la règle générale. Elle a été prévue et, dans la majorité des cas, réalisée lors de la construction des ouvrages principaux (la machine de Pierre-Bénite a fait l'objet d'une installation différée dans une pile aménagée d'origine). Les installations projetées sur le Bas-Rhône échapperont évidemment à cette règle.

Les grilles de prise d'eau équipées d'un dégrilleur sommaire (sans poche) sont situées dans le mur d'entonnement amont du barrage. Cette disposition présente en particulier l'avantage de bien se prêter à l'élimination des corps flottants par ouverture du clapet de la passe de rive.

(3) Y compris les sites existants. 


\section{débit réservé $\left[\mathrm{m}^{3} / \mathrm{s}\right)$}

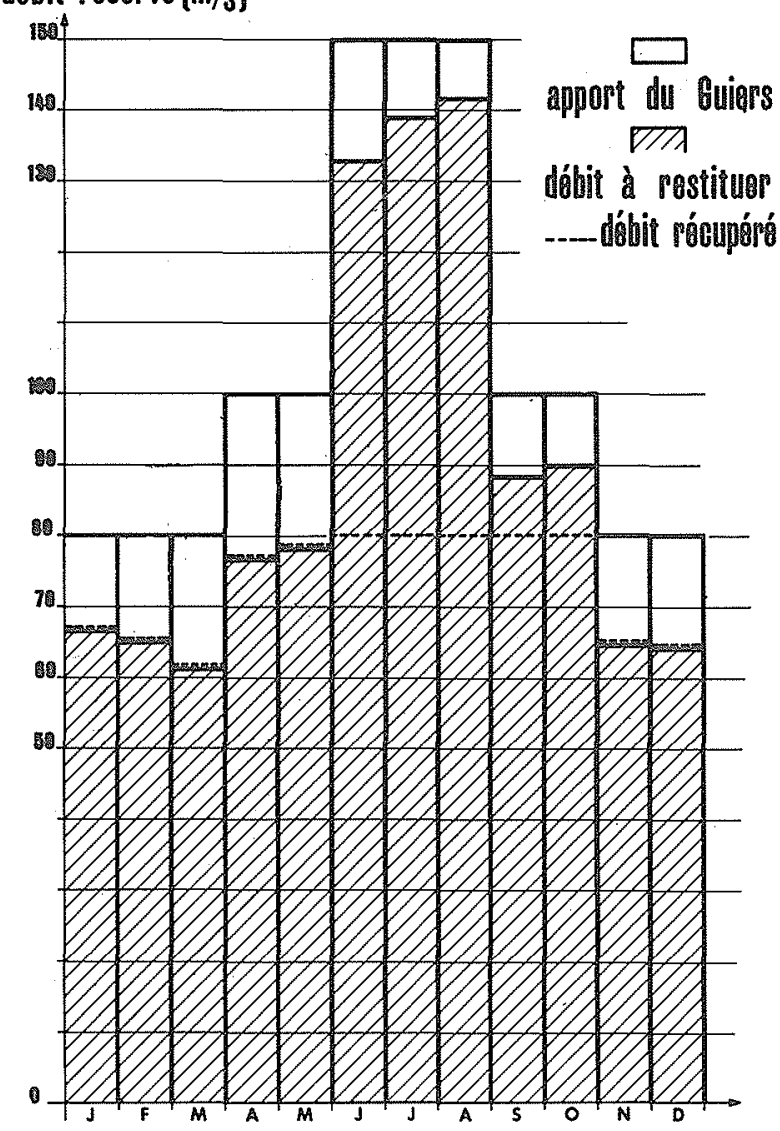

Figure 6 - Bregnier Cordon -- Consigne de débit réservé.

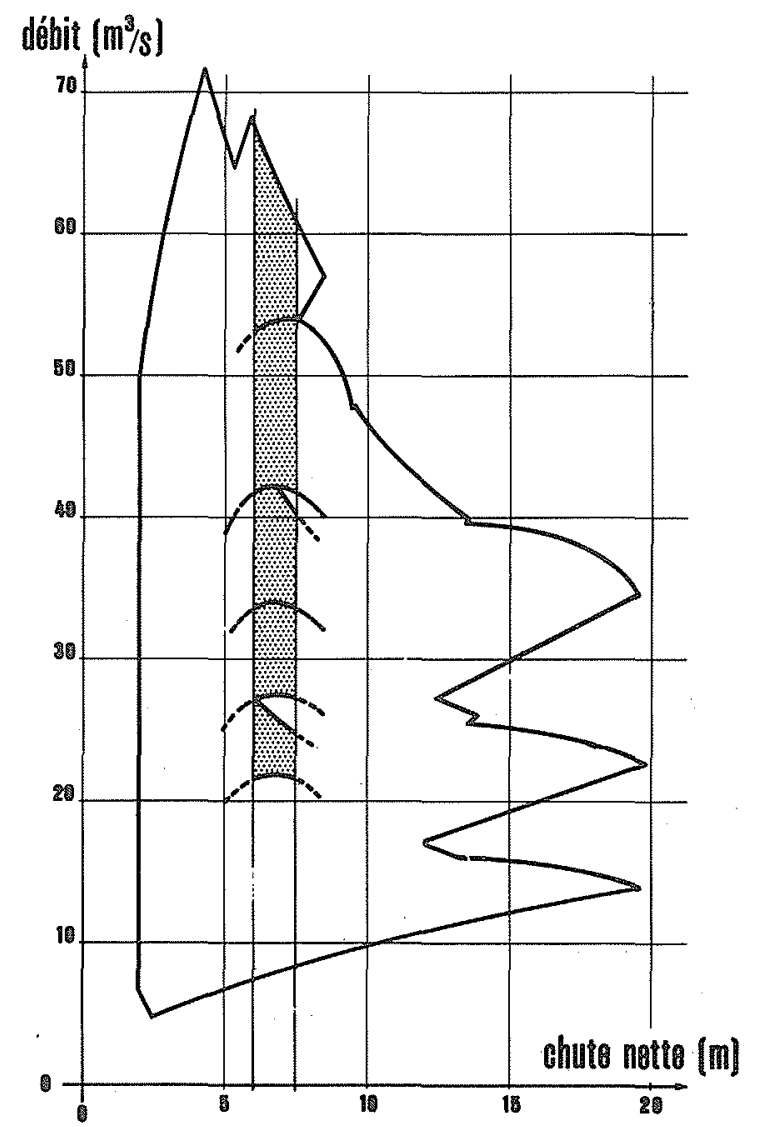

Figure 7 - Bregnier Cordon - Groupes "bulbe" normalisés NEYRPIC - zones d'utilisation.

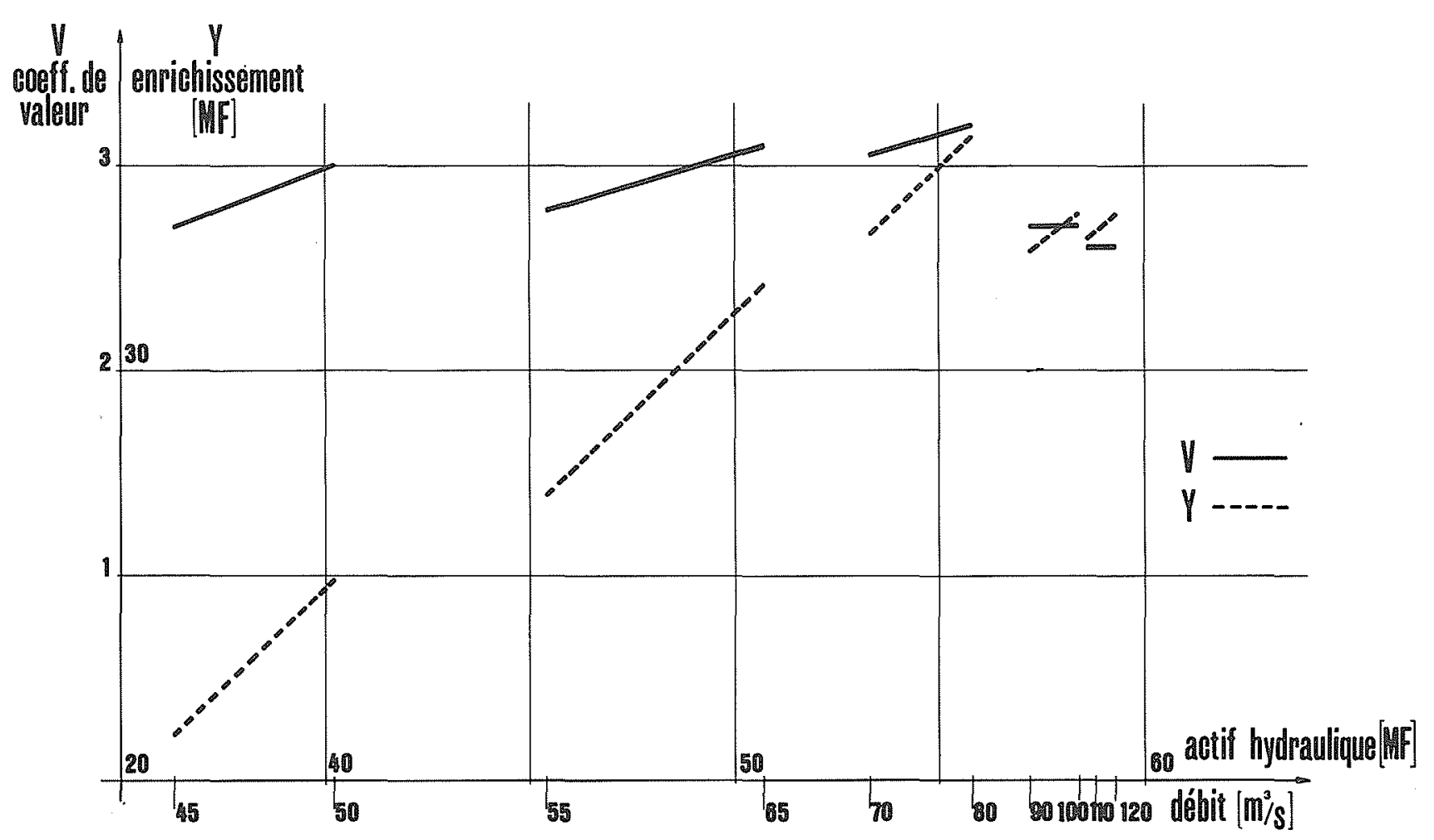

Figure 8 - Bregnier Cordon - Résultats de l'étude économique. 


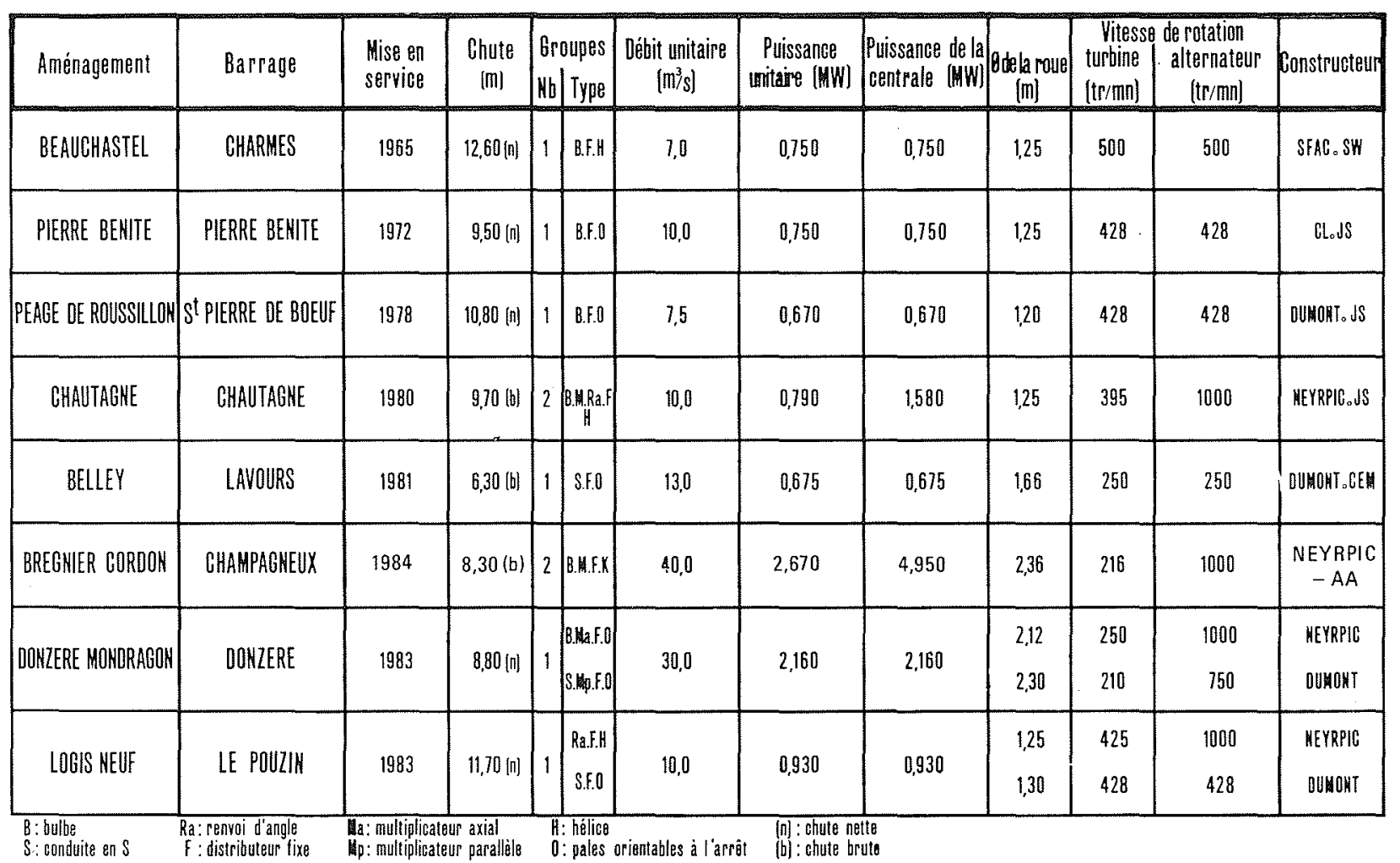

Figure 9 - Tableau récapitulatif des installations de récupération du Rhône.

\section{Charmes (Beauchastel), Pierre-Bénite et St-Pierre de Boeuf (Péage de Roussillon)}

Ces installations appartiennent à une première génération, caractérisée par une consigne de débit réservé, simple et appliquée à l'aval immédiat du barrage .

Elles ont conduit à la mise en œuvre des groupes bulbes "amont" à entraînement direct d'un alternateur lent, avec des technologies diverses. Leur fonctionnement est sûr, seul le groupe de Charmes ayant donné lieu dans ses débuts d'exploitation à des interventions pour remise en état des étanchéités d'arbre et réparation d'usures de cavitation. Toutefois, pour ces petites puissances, ces solutions à génératrice lente immergée sont d'un prix relativement élevé.

\section{Chautagne (Fig. 10).}

La consigne de débit réservé est du même type que précédemment mais l'évolution du marché des petites machines conduit à envisager des solutions nouvelles moins coûteuses.

Les deux groupes comportent une turbine sans réglage qui entraine, par l'intermédiaire d'un multiplicateur à pignons coniques, une génératrice rapide située à l'extérieur du conduit.

Le faible encombrement des machines permet de les insérer côte à côte dans une même pile-culée du barrage, légèrement agrandie.

Les turbines sont livrées entièrement montées. Le portique à batardeaux du barrage est utilisé pour assurer leur mise en place en fond de fosse où elles sont positionnées puis scellées dans des réservations du béton de première phase.

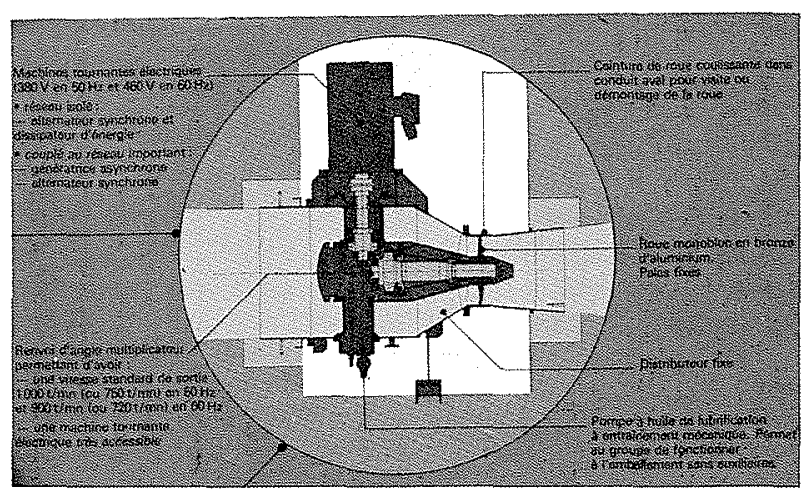

Figure 10 - Chautagne - Coupe longitudinale d'un groupe à renvoi d'angle.

\section{Belley}

La consigne de débit réservé est complexe. L'optimisation de l'équipement a conduit à choisir un groupe fixe de $13 \mathrm{~m}^{3} / \mathrm{s}$ qui sera installé dans la pile-culée Rive Droite mais les incertitudes qui affectent la détermination des apports intermédiaires, nous ont amenés à prévoir la pile Rive Gauche à l'identique, de façon à ménager la possibilité de compléter, si nécessaire, l'installation actuelle.

Avec celles de Lamanon et Strasbourg, la machine de I lley présente une architecture en " $S$ " qui permet, con....e la solution "à renvoi d'angle" choisie pour Chautagne, de sortir la génératrice du conduit, ici, vers l'amont. Cette disposition autorise l'utilisation 
d'un aspirateur droit. L'interposition d'un multiplicateur est possible dans cette technique, mais n'a pas été envisagée dans le cas de Belley.

\section{Brégnier-Cordon}

Le débit équipé est approximativement 10 fois celui de Saint-Pierre de Bœuf, il en résulte un changement de taille sensible. L'implantation du matériel présente, de ce fait, par rapport aux installations antérieures, diverses particularités:

- les deux groupes sont abrités par une petite usine accolée au barrage. Ce bâtiment, contigu à la pile-culée Rive Droite, abrite les auxiliaires électriques du barrage et des groupes (groupe thermique de secours, alimentations diverses, batteries de condensateurs, etc.) ;

- les sorties des aspirateurs, dont la côte de calage n'est pas compatible avec celle du radier de la passe adjacente, sont frontales et séparées de cette passe par un mur divisoir ;

- pour les groupes, et compte tenu des variations de débit attendues, la solution retenue comporte une turbine à pales réglables en marche. Sans doute un peu plus contraignante au point de vue des travaux d'entretien, que la solution sans réglage, elle ne devrait, en revanche, pas alourdir les tâches de conduite. En effet, l'automate qui commande les vannes et volets du barrage gère aussi les groupes de récupération.

\section{Projets sur le Bas-Rhône}

Leur équipement n'est pas encore définitivement arrêté, mais il est intéressant de souligner à leur sujet, les difficultés d'implantation propres aux aménagements déjà en service, du fait que le génie civil n'a pas été prévu dès l'origine. On peut affirmer que les solutions techniques ne manquent pas mais les dépenses à consentir grèvent lourdement le bilan économique de lopération.

Les différentes réalisations et projets qui viennent d'être décrits, donnent, pour ce qui concerne les plus récents, une physionomie des solutions disponibles actuellement : groupes à renvoi d'angle, groupes en "S" avec leurs différentes variantes amont, aval et groupe bulbe à multiplicateur planétaire. Il faut signaler encore deux solutions qui peuvent être introduites dans les études de faisabilité : il s'agit des groupes "puits" et des groupes avec alternateur annulaire (ou périphérique). Ce dernier type de machine a été retenu récemment dans sa version la plus courante, c'est-à-dire sans réglage de débit, pour l'équipement de chutes sur la Meuse, en Belgique.

$\mathrm{Si}$ on excepte les machines à renvoi d'angle pour lesquelles nous connaissons peu d'antécédents dans le domaine hydroélectrique (cette technologie est dérivée de propulseurs marins) les différentes architectures citées, présentent en commun la caractéristique de n'être pas nouvelles.

Sous la double influence de la demande croissante (crise énergétique, électrification dispersée dans les pays en voie de développement) et du progrès technique, il semble que les constructeurs aient pris le parti, pour répondre à la grande diversité des situations rencontrées, de réexaminer les solutions essayées puis abandonnées ou maintenues au cours des cinquante dernières années.

\section{Abstract \\ Recovery of hydraulic energy: small installations associated with major hydraulic projects}

Small hydroelectric installations associated with major projects are covered, in France, by the same legal regime as pure hydroelectric installations.

The hydraulic link with the larger structures usually takes one of two forms:

1) the sets transfer or recover a reserve flow which is restored to the river;

2) the sets are installed on existing structures built for purposes other than the production of energy.

The reassessments of energy value resulting from the successive oil crises have led to justification of the energy benefit provided by new installations linked to existing hydraulic equipment; the projects of Electricité de France and Compagnie Nationale du Rhône represent a production of 280 millions $\mathrm{kWh}$ and a power of nearly $50,000 \mathrm{~kW}$.

For Electricité de France in particular, various examples of developments are presented, namely:
- the return plant at Nèpes, downstream of the St. Etienne Cantalès dam $(2,8 \mathrm{MW}, 8 \mathrm{GWh})$;

- the Lamanon plant (1.6 MW, 8.7 GWh);

- the Strasbourg return plant ( $1 \mathrm{MW}, 8 \mathrm{GWh})$;

- the project for equipping the navigation dams on the Moselle (32 MW, $180 \mathrm{GWh}$ ).

For its part, Compagnie Nationale du Rhône contributes some thoughts on the dimensioning of the small machines used to equip the recovery installations for the low-head barrages on the Rhône.

The existing installations mentioned are: Charmes, Pierre Benite, and St. Pierre de Bceuf.

Others being built or projected are described:

Chautagne: $(2$ sets) $-1.6 \mathrm{MW}-9.6 \mathrm{GWh} /$ year

Belley: (1 set) $-0.7 \mathrm{MW}-4.8 \mathrm{GWh} /$ year

Bregnier Cordon: (2 sets) - 4.4 MW - 33.8 GWh/year

Projects concerning certain developments already being operated are also mentioned. 


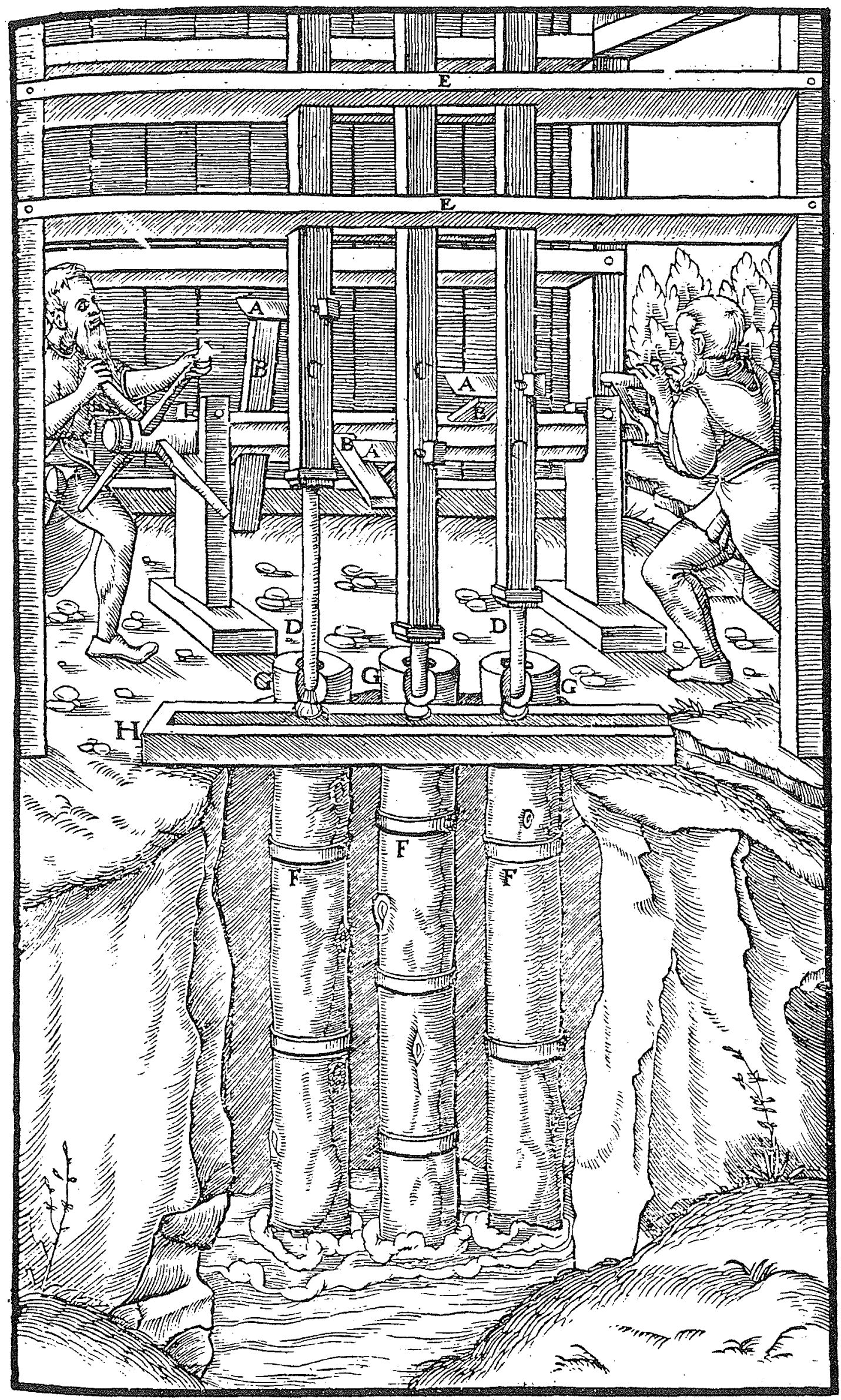

